\title{
Multiple topological phase transitions in a gyromagnetic photonic crystal
}

\author{
Ze-Guo Chen, ${ }^{1}$ Jun Mei, ${ }^{2, *}$ Xiao-Cheng Sun, ${ }^{3}$ Xiujuan Zhang, ${ }^{1}$ Jiajun Zhao, ${ }^{1}$ and Ying $\mathrm{Wu}^{1, \dagger}$ \\ ${ }^{1}$ King Abdullah University of Science and Technology (KAUST), Division of Computer, \\ Electrical and Mathematical Science and Engineering (CEMSE), Thuwal 23955-6900, Saudi Arabia \\ ${ }^{2}$ Department of Physics, South China University of Technology, Guangzhou 510640, China \\ ${ }^{3}$ Department of Materials Science and Engineering, National Laboratory of Solid State Microstructures, \\ Nanjing University, Nanjing 210093, China
}

(Received 30 December 2016; published 18 April 2017)

\begin{abstract}
We present the design of a tunable two-dimensional photonic crystal that exhibits multiple topological phases, including a conventional insulator phase, a quantum spin Hall phase, and a quantum anomalous Hall phase under different combinations of geometric parameters and external magnetic fields. Our photonic crystal enables a platform to study the topology evolution attributed to the interplay between crystalline symmetry and time-reversal symmetry. A four-band tight-binding model unambiguously reveals that the topological property is associated with the pseudospin orientations and that it is characterized by the spin Chern number. The emerging quantum anomalous Hall phase features a single helical edge state that is locked by a specific pseudospin. Simulation results demonstrate that the propagation of such a single helical edge state is robust against magnetic impurities. Potential applications, such as spin splitters, are described.
\end{abstract}

DOI: 10.1103/PhysRevA.95.043827

\section{INTRODUCTION}

A key principle of the quantum Hall $(\mathrm{QH})$ effect is the presence of a magnetic field that breaks the time-reversal (TR) symmetry, leading to quantized Hall conductance and the chiral edge states of electrons [1-3]. Quantized Hall conductance is associated with the Chern number, a mathematical concept that characterizes the topology of the electronic bands. Recently, a new class of topological effect, namely, the quantum spin Hall (QSH) effect [4-6], was predicted in the two-dimensional (2D) Kane-Mele model [7] and confirmed in HgTe quantum wells [8,9]. Different from the QH effect, the QSH effect is attributed to the spin-orbit coupling that preserves TR symmetry. The QSH effect gives rise to helical edge states [10] in which opposite spins counterpropagate and contribute to opposite quantized Hall conductivities. The chiral and helical edge states, respectively, produce robust charge and spin charge edge currents protected by the bulk topology of the system, implying promising possibilities for spintronics and quantum computing [11].

TR symmetry plays a subtle role in the robustness of the QSH effect [12-14]. For Fermi particles, such as electrons, preserved TR symmetry guarantees Kramers' degeneracy and associates two oppositely moving edge states with opposite spins. Elastic backscattering is thus forbidden under any nonmagnetic scatterer that preserves TR symmetry. Slightly breaking the TR symmetry in a QSH sample leads to a TR-broken QSH phase in which the states associated with different spins are separated without changing their propagating directions [15]. Further breaking the TR symmetry results in a quantum anomalous Hall (QAH) phase [16] in which only one helical edge state locked by a particular moving direction (or spin orientation) survives on the boundary $[17,18]$. Due to the well-known difficulties in realizing the

\footnotetext{
*phjunmei@ scut.edu.cn

†ying.wu@kaust.edu.sa
}

sophisticated Hamiltonian, the experimental demonstration of the TR-broken-QSH effect in the electronic system is limited.

Recently, the study of the $\mathrm{QH}$ effect and chiral edge states was extended successfully to classical wave systems due to the analogy between the electronic and the photonic or phononic band gaps [13,19-23]. However, the extension of the QSH effect was more challenging because fermions and bosons are radically different under the time-reversal operation. Despite this difficulty, many groups have observed that Kramers' degeneracy and the associated QSH effect can also be achieved for pseudospin-1/2 states in systems where pseudo-TR symmetry is constructed from particular spatial symmetries [24-34]. Pioneering examples of constructions of pseudospin-1/2 states include the use of hybrid transverse electric (TE) + transverse magnetic (TM) and TE-TM states [26,28,29], left circular polarization and right circular polarization states, or clockwise and counterclockwise helical energy flow states [24,25,30,33]. It has been proved that applying the pseudo-TR operator on a pseudospin-1/2 state is mathematically equivalent to applying the real TR operator on a real spin-1/2 state. Given these observations, we wondered what the consequence would be when real $T R$ symmetry is broken in a QSH sample that possesses pseudo-TR symmetry. The answer to this question should provide more profound understanding of the band topology in both classical and quantum systems because these two types of systems share similar mathematical foundations. The seemly difficult problem in quantum systems may be resolved in classical systems as more degrees of freedom are offered in the engineering of the band topology.

In this paper, we seek an answer to the question by analyzing the topological properties of a simple 2D photonic crystal (PC) made from a gyromagnetic material. Without an external magnetic field, such a 2D PC may behave either as a QSH insulator with pseudo-TR symmetry or as a conventional insulator, depending on the geometry. We found that, by applying a gradually enhanced external magnetic field, the 2D PC goes through either a phase transition from a QSH 

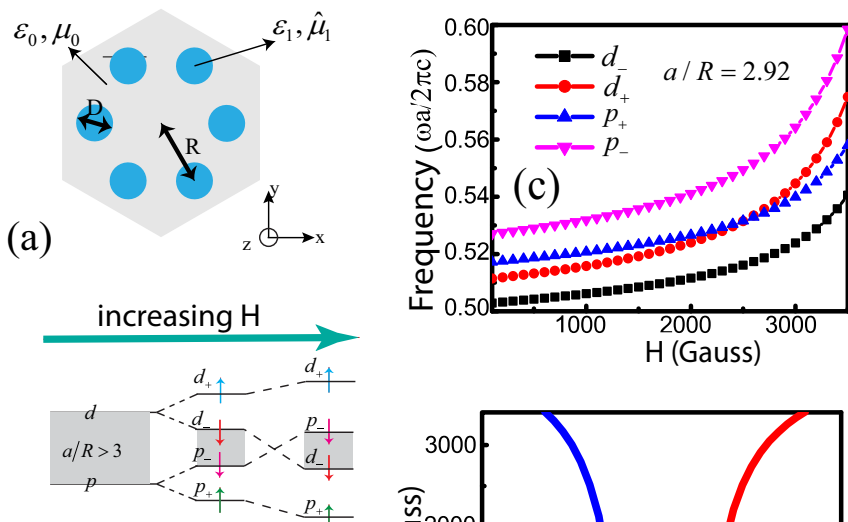

(b)
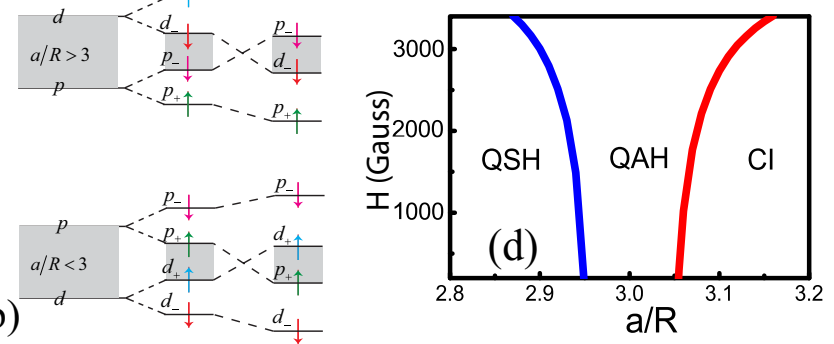

FIG. 1. Construction of a photonic crystal supporting the topological transition from a conventional insulator (or a QSH insulator) to a QAH insulator. (a) Schematic of a unit cell. Parameter $R$ is tunable, and the permeability tensor $\hat{\mu}_{1}$ can also be tuned under a dc magnetic field. (b) Evolution of the eigenstates under an increasing external magnetic-field $H$. The gray area indicates a band gap. There are two initial phases: A conventional insulator when $a / R>3$ and a QSH insulator when $a / R<3$. Increasing $H$ lifts the degeneracies of the pseudospins, resulting in a gap closing and reopening. (c) Frequencies of the eigenstates as functions of the applied external magnetic field when $a / R=2.92$. (d) A phase diagram of the topological property with combined modulation of $R$ and $H$. The left blue (right red) line indicates the topological transition between the QSH insulator (conventional insulator) and the QAH insulator. Note that $H$ starts from $100 \mathrm{G}$, which is strong enough for the magnetization of the yttrium iron garnet (YIG) material to reach the saturated state.

insulator to a TR-broken-QSH insulator and then to a QAH insulator or a transition from a conventional insulator to a QAH insulator. If the external magnetic field is fixed, gradually increasing the distance between the neighboring cylinders in a unit cell may result in a phase transition from a conventional insulator to a QAH insulator and then to a TR-broken-QSH insulator. To capture the essence of these phase transitions, we developed an effective Hamiltonian and classified the topological properties by the spin Chern number, a topological invariant that is uniquely defined in different phases [35]. Both our theoretical model and numerical simulations show the existence of a tunable topological edge state locked by a particular pseudospin orientation.

\section{PHOTONIC CRYSTAL AND TOPOLOGICAL PHASES}

The 2D PC considered here is a triangular lattice of hexagonal clusters. The lattice constant, i.e., the distance between the centers of the neighboring clusters, is $a$. A hexagonal cluster (unit cell) is illustrated in Fig. 1(a), which shows six identical cylinders made from the gyromagnetic material yttrium iron garnet (YIG, $\varepsilon_{1}=15 \varepsilon_{0}$ ) and distributed in air. $\varepsilon_{0}$ is the dielectric constant of air. The distance between the center of a cluster to the center of its associated cylinder is
$R$, which can be adjusted. The diameter of each cylinder is set to $D / a=0.17$. The permeability tensor $\hat{\mu}_{1}$ of YIG depends on the applied magnetic field and frequency, whose detailed expressions are obtained from the Drude model [36].

We start from the eigenvalue problem of an out-of-plane wave $\left[\vec{E}=\left(0,0, E_{z}\right)\right]$ governed by Maxwell's equation in our design of a PC [3]. Without the external magnetic field, the PC possesses two doubly degenerate eigenstates in the center of the Brillouin zone. These eigenstates are associated with two 2D irreducible representations of a $C_{6 v}$ point group, i.e., $E_{1}$ and $E_{2}$, and share the same symmetry with the basis functions of them. The basis functions can be classified according to their spatial parities, marked as $p_{x}, p_{y}, d_{x y}$, and $d_{x^{2}-y^{2}}$, a conventional notation widely adopted to describe electronic orbitals. The $p_{x}$ and $p_{y}$ states degenerate and so do the $d_{x y}$ and $d_{x^{2}-y^{2}}$ states. There exists a band gap between the $p$ states and the $d$ states. It was demonstrated in Ref. [30] that these states can form two pseudospin states $\left(p_{ \pm}=p_{x} \pm i p_{y}\right.$ and $d_{ \pm}=$ $d_{x y} \pm i d_{x^{2}-y^{2}}$ ) that can be recognized from the patterns of the Poynting vectors $\vec{S}=\operatorname{Re}\left[\vec{E} \times \vec{H}^{*}\right] / 2$. The Poynting vectors circle around in a unit cell, and the counterclockwise (clockwise) rotation corresponds to the pseudospin-up (pseudospindown) state. These states form a basis $\left(\left[p_{+}, d_{+}, p_{-}, d_{-}\right]\right)$upon which a tight-binding model is developed. The Hamiltonian near the $\Gamma$ point is written as $H_{0}=E_{0}+D k^{2}+A\left(\hat{\tau}_{z} k_{y} \hat{\sigma}_{x}-\right.$ $\left.k_{x} \hat{\sigma}_{y}\right)+\left(M_{0}-B k^{2}\right) \hat{\sigma}_{z}$, where $E_{0}, A, D, M_{0}$, and $B$ are model parameters [37] and are described in detail in Appendix A. $\hat{\tau}$ and $\hat{\sigma}$ are the Pauli matrices for the two pseudospins and the two bands, respectively.

The external magnetic field separates the degenerate pseudospin states. The magnetic field is modeled by an effective vector potential [3], resulting in the split of the spinlike Zeeman-type spin splitting. We introduce a perturbation Hamiltonian [38,39],

$$
H_{z}=\left(\begin{array}{cccc}
z_{p} & 0 & 0 & 0 \\
0 & z_{d} & 0 & 0 \\
0 & 0 & -z_{p} & 0 \\
0 & 0 & 0 & -z_{d}
\end{array}\right)+z_{0} I,
$$

where the pseudospin splitting is $2 z_{p}$ for the $p_{ \pm}$states and $2 z_{d}$ for the $d_{ \pm}$states. $z_{0}$ is the total energy shift caused by the gyromagnetic material under an applied magnetic field, which does not affect the topology of the bands. Since the "effective masses" for the $p$ band and the $d$ band have opposite signs, we have $z_{p} z_{d}<0$ (here $z_{p}<0, z_{d}>0$ ). The detailed expressions of the Hamiltonian with an external magnetic field are presented in Appendix A.

Figure 1(b) shows the evolution of the eigenfrequencies of the originally degenerate pseudospin states under the excitation of an external magnetic field. Two initial states associated with different geometries are considered. To characterize the topology of the band, we employ the theory of the spin Chern number [40] and derived the following expression (details are available in Appendix B):

$$
C_{ \pm}= \pm\left[\operatorname{sgn}(B)+\operatorname{sgn}\left(M_{0} \pm g\right)\right] / 2,
$$

where $g=\left(z_{p}-z_{d}\right) / 2<0$ in our photonic system. When $g=0$, the system exhibits a topological transition from a conventional insulator to a QSH insulator by simply increasing 

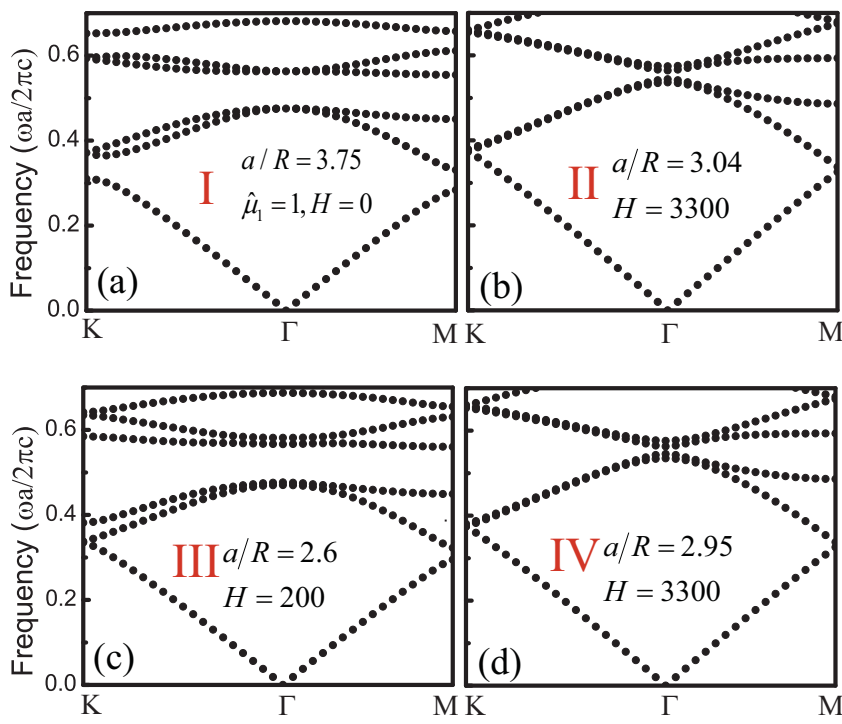

FIG. 2. Bulk bands of four PCs with a common global band gap. (a) System I with $a / R=3.75$, and $H=0$ corresponds to a conventional insulator. (b) System II with $a / R=3.04$, and $H=$ $3300 \mathrm{G}$ corresponds to a QAH insulator. (c) System III with $a / R=$ 2.6, and $H=200 \mathrm{G}$ corresponds to a TR-broken-QSH insulator. (d) The same as (b) but $a / R=2.95$.

$R$. For example, without the external magnetic field, the system is a QSH insulator $\left(M_{0}>0, B>0\right)$ with spin Chern number $C_{ \pm}=(1,-1)$ when $a / R<3$; but it is a conventional insulator $\left(M_{0}<0, B>0\right)$ with spin Chern number $C_{ \pm}=0$ when $a / R>3$. These two cases correspond to the initial states shown in Fig. 1(b), which were extensively discussed in Ref. [30]. It is noted that these two initial phases can also be characterized by the $\mathbb{Z}_{2}$ index since they respect the TRS. Once the external magnetic field is applied, the $\mathbb{Z}_{2}$ index is not valid, and the spin Chern number has been proved to be the topological invariant [35]. In this case, the $g$ in Eq. (1) is no longer zero, and its magnitude increases as the applied field increases. When the magnitude of $g$ is greater than $M_{0}$, the spin Chern number becomes $C_{ \pm}=(0,-1)$, and the system becomes a QAH insulator regardless of the initial state. The topological transition from a QSH phase (or a conventional insulator phase) to a QAH phase is associated with the band inversion of $p_{+}$and $d_{+}$(or $p_{-}$and $d_{-}$) shown in Fig. 1(c) and can be predicted numerically from the evolution of the eigenfrequencies of the relevant states at the $\Gamma$ point. Figure 1(d) presents the topological phase diagram as a function of the geometric parameters and the external magnetic field. Each point on the curve indicates a Dirac cone at the $\Gamma$ point. These cones are attributed to the accidental degeneracy of the $p_{+}$and $d_{+}$states (red) or the $p_{-}$and $d_{-}$ states (blue). Topological transitions between QSH and QAH phases occur across the blue curve. Similarly, the topological transition between QAH and conventional insulator phases happens on the red curve.

Figure 2 shows four representative band structures of the photonic crystal with different geometric parameters and external magnetic fields. The results are calculated by the commercial software COMSOL MULTIPHYSICS under the

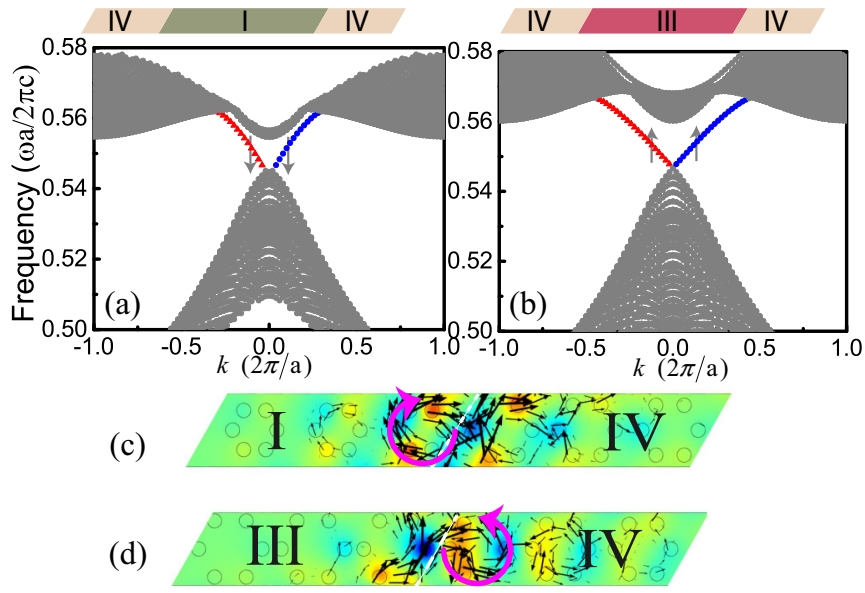

FIG. 3. Projected band structures and edge states. (a) Dispersion relation for a ribbon-shaped 2D topological PC with 80 unit cells formed by systems I and IV. Red triangles (blue circles) indicate the edge state in the left (right) interface. The gray arrows represent the pseudospins. (b) The same as (a) but for systems III and IV. (c) Distributions of the $E_{z}$ field at $k=0.4 \pi / a$ with clockwise Poynting vectors. (d) Edge states with counterclockwise Poynting vectors between systems III and IV.

assumption that the parameters $\varepsilon_{1}$ and $\hat{\mu}_{1}$ are not sensitive to frequency. This assumption is valid around $14.5 \mathrm{GHz}$. We set the lattice constant to be $a=1.15 \mathrm{~cm}$ in our simulations for the sake of producing a common band gap for various scenarios in our later analysis. In Fig. 2, the upper (lower) panel corresponds to two cases with $a / R>3(a / R<3)$. The respective external magnetic fields are marked in each plot. All scenarios share a common band gap around the dimensionless frequency $\omega a /(2 \pi c)=0.5541$. According to our previous analysis as shown in Fig. 1(c), system I is a conventional insulator with $C_{ \pm}=0$, systems II and IV are QAH insulators with the same topological invariant $C_{ \pm}=(0,-1)$, and system III is a TR-broken-QSH insulator with $C_{ \pm}=(1,-1)$. We note that the external magnetic field applied on system III is weak, meaning the TR symmetry is broken slightly.

\section{EDGE STATES}

Overlapping the band gaps of different types of insulators benefits the study of edge states on the interfaces between insulators with different spin Chern numbers. We construct two supercells, one from a conventional insulator (system I) sandwiched between two identical QAH insulators (system IV), and the other a broken TR-QSH insulator (system III) in a QAH insulator (system IV). As illustrated in Figs. 3(a) and 3(b), both supercells contain 80 unit cells divided equally into different insulators. Each supercell contains two interfaces. The calculated projected band structures are plotted in Figs. 3(a) and 3(b) where the red and blue lines indicate the edge states on the left and right interfaces, respectively. For the case shown in Fig. 3(a), systems I and IV carry spin Chern numbers $C_{ \pm}=0$ and $C_{ \pm}=(0,-1)$, respectively. For the pseudospin-up $(+)$ component, the entire system is topologically equivalent, but for the pseudospin-down (-) component, it behaves like a $\mathrm{QH}$ system characterized by a 

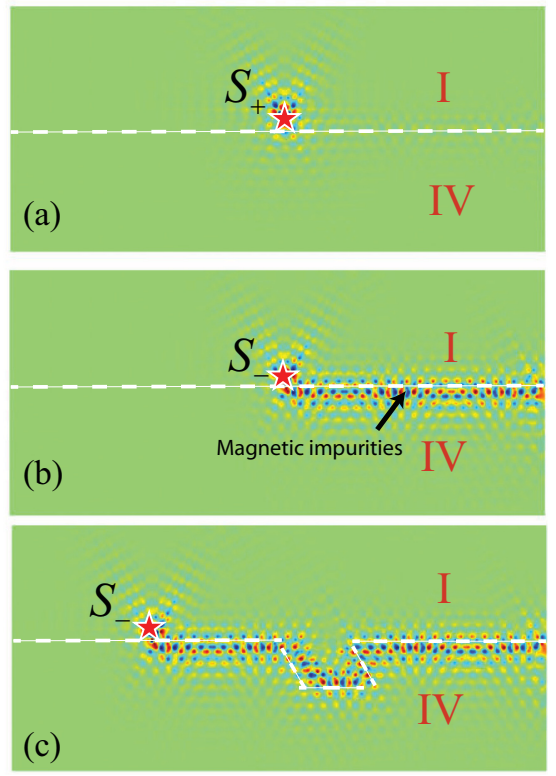

FIG. 4. Single helical edge state and robust one-way propagation. The source is marked as a star with operating frequency $0.5533 \omega c /(2 \pi a) . S_{+}\left(S_{-}\right)$represents pseudospin-up (pseudospindown), which is excited by in-plane magnetic fields with a $\pi / 2$ phase difference: $S_{ \pm}=H_{0}(\hat{x} \mp i \hat{y})$. (a) Conventional insulator behavior when excited by a pseudospin-up source. (b) Robust one-way propagation of a helical state against magnetic impurities with a pseudospin-down source excitation. (c) Robust one-way propagation of a helical state against sharp bends.

gapless edge state as shown in Fig. 3(a). We find that the edge states are located at the interface and decay exponentially into the bulk [displayed in Fig. 3(c)]. The edge state exhibits clockwise rotation behavior in the Poynting vector, indicating that the state is associated with a pseudospin-down state, which verifies our prediction. A similar argument can be extended to the case shown in Fig. 3(b) in which the spin Chern numbers are $C_{ \pm}=(1,-1)$ for system III and $C_{ \pm}=(0,-1)$ for system IV. Although spin Chern numbers for the pseudospin-down component in both systems are nonzero, they are identical. Thus, there exists no edge state associated with the pseudospin-down component. The difference in the spin Chern numbers for the pseudospin-up component guarantees the existence of the edge state as shown in Figs. 3(b) and 3(d). The counterclockwise pattern of the Poynting vector shown in Fig. 3(d) coincides with the characteristic of a pseudospin-up state.

The pseudospin-dependent feature also is manifested in our full wave simulations of a finite $(40 \times 20)$ lattice as shown in Fig. 4 in which the upper half of each panel shows system I and the lower half shows system IV. When excited by a pseudospin-up source, the entire system behaves like a conventional insulator, and there exists no edge state [Fig. 4(a)]. On the contrary, if the source contains only a pseudospin-down component, one-way propagation edge states emerge as displayed in Fig. 4(b). This behavior is distinct from that in QSH insulators which are characterized by pairs of helical edge states.

The robustness of the edge states is demonstrated by introducing a magnetic impurity or geometric deformations

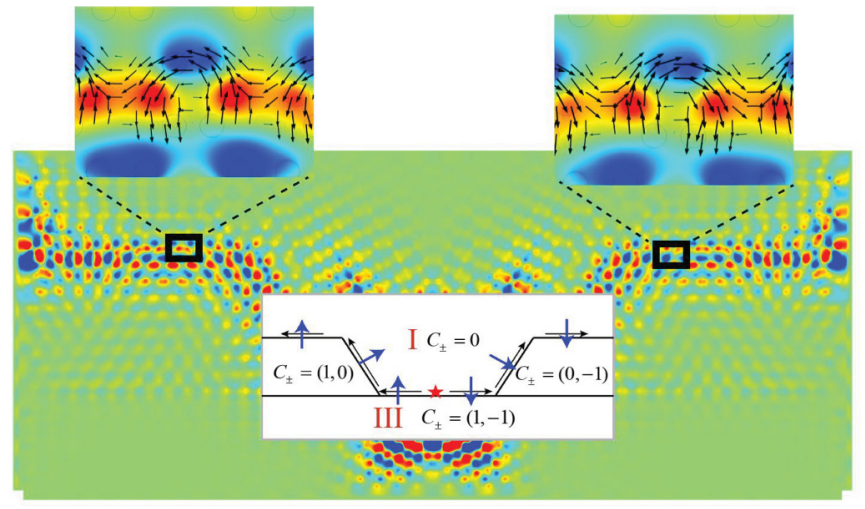

FIG. 5. A photonic pseudospin splitter. The insets: schematic of the pseudospin splitter. The star indicates an out-of-plane $E_{z}$ source. The black arrows indicate the propagation directions of the edge states, whose pseudospins are represented by blue arrows. The structure $(40 \times 20$ lattices $)$ is surrounded by a perfectly matched layer. The operating frequency is the same as that in Fig. 4. The enlarged field patterns show the pseudospin splitting effect in which the arrows denote the Poynting vectors (pseudospin orientations).

on the edge. The magnetic impurity is caused by replacing the cylinders in one unit cell with different permeability tensors [Fig. 4(b)], and geometric deformations are constructed by replacing a parallelogram region of system IV with that of system I [Fig. 4(c)]. Both Figs. 4(b) and 4(c) clearly exhibit the defect-immune one-way propagation behavior of pseudospin-down edge states. It should be noted that the robustness of helical edge states in QSH insulators is protected by TR symmetry. Thus, introducing a magnetic impurity would cause backscattering in a QSH insulator. In our system, due to the lattice deformation and the applied magnetic field, both pseudo-TR symmetry and real TR symmetry are broken. However, the single helical edge state is protected by the topological invariant and spin Chern number, and therefore no backscattering occurs even with the presence of magnetic impurities. This striking difference distinguishes our system from conventional QSH insulators. Similar results can be obtained for the pseudospin-up component along the interface between systems III and IV. We also want to emphasize that the one-way propagation edge states here are associated with the pseudospin states which require the $C_{6}$ lattice symmetry in 2D. It is impossible to achieve such a kind of pseudospin-locked edge state in a PC with $C_{4}$ symmetry.

The robust pseudospin-dependent propagation against magnetic impurities has several potential applications. Figure 5 shows that the waves emanating from a trivial source (a line source with an out-of-plane $E_{z}$ field) can be divided into separate pseudospin-up and pseudospin-down components in a carefully designed sample. We insert two QAH insulators into the interface of a conventional insulator (system I) and a TR-broken-QSH insulator (system III). The geometric parameters of these two QAH insulators are identical to those of insulator IV, but the applied magnetic field is in the opposite direction. Both theoretical analysis and numerical simulation demonstrate the robust one-way propagation behavior of particular spin orientations. The existence of the two QAH 
insulators ensures that the edge states are robust against both magnetic and nonmagnetic defects.

\section{CONCLUSION}

In conclusion, we propose a tunable photonic crystal with multiple topological phases. It can behave as a conventional insulator, a QSH insulator, or a QAH insulator by simply adjusting the geometric parameters and/or the magnetic field. A four-band tight-binding model clearly captures the phase transitions between different phases and reveals the subtle relation between the pseudo-TR symmetry and the real TR symmetry. It unambiguously shows that the topological invariant of the band is the spin Chern number and predicts the existence of a robust single helical edge state that is associated with pseudospin. The prediction is verified by numerical simulations, implying potential applications, such as spin splitter devices.

\section{ACKNOWLEDGMENTS}

The work described here was supported by King Abdullah University of Science and Technology and the National Natural Science Foundation of China (Grants No. 11274120 and No. 11574087).

\section{APPENDIX A: HAMILTONIAN WITHOUT AND WITH AN APPLIED MAGNETIC FIELD}

In this appendix, we derive the Hamiltonian based on the tight-binding method. The system we studied requires a fourband model containing four basis states classified by different symmetries. The total energy is given in the language of second quantization: $\hat{H}_{0}=\sum_{\hat{m} \hat{n} \alpha \beta} t_{\hat{m} \hat{n}}^{\alpha \beta} c_{\hat{n} \beta}$, where $\hat{m}$ and $\hat{n}$ are indices of the lattice sites and $\alpha$ and $\beta$ take the values (1-4), which correspond to the states $\left(p_{x}, p_{y}, d_{x y}\right.$, and $\left.d_{x^{2}-y^{2}}\right)$, respectively. $t_{\hat{m} \hat{n}}^{\alpha \beta}$ describes the coupling between state $\alpha$ on site $\hat{m}$ and state $\beta$ on site $\hat{n}$. On the conditions that only the nearest coupling is considered and that the periodic boundary condition is invoked and after taking the Fourier transform, we obtain the Hamiltonian $\hat{H}_{0}(\mathbf{k})$, whose entries are $\hat{H}_{\alpha \beta}(\mathbf{k})=\sum_{\mathbf{r}=0}^{6} t_{\mathbf{r}}^{\alpha \beta} e^{-i \mathbf{k} \cdot \mathbf{r}}$. Here, $\mathbf{r}$ represents the relative locations of the neighboring unit cells. The $\mathbf{r}=0$ term corresponds to the coupling of a unit cell with itself. By fully exploiting the $C_{6 v}$ symmetry, we found that there are eight independent coupling coefficients, and the resulting Hamiltonian can be written as [37]

$$
\hat{H}_{0}(\mathbf{k})=\left(\begin{array}{cccc}
H_{11} & H_{12} & H_{13} & H_{14} \\
H_{12}^{*} & H_{22} & H_{23} & -H_{13} \\
H_{13}^{*} & H_{23}^{*} & H_{33} & H_{34} \\
H_{14}^{*} & -H_{13}^{*} & H_{34}^{*} & H_{44}
\end{array}\right),
$$

where

$$
\begin{aligned}
H_{11}= & t_{0}^{11}+2 t_{1}^{11} \cos \left(k_{x}\right) \\
& +\left(t_{1}^{11}+3 t_{1}^{22}\right) \cos \left(\frac{k_{x}}{2}\right) \cos \left(\frac{\sqrt{3} k_{y}}{2}\right), \\
H_{22}= & t_{0}^{22}+2 t_{1}^{22} \cos \left(k_{x}\right) \\
& +\left(3 t_{1}^{11}+t_{1}^{22}\right) \cos \left(\frac{k_{x}}{2}\right) \cos \left(\frac{\sqrt{3} k_{y}}{2}\right),
\end{aligned}
$$

$$
\begin{aligned}
H_{33}= & t_{0}^{33}+2 t_{1}^{33} \cos \left(k_{x}\right) \\
& +\left(t_{1}^{33}+3 t_{1}^{44}\right) \cos \left(\frac{k_{x}}{2}\right) \cos \left(\frac{\sqrt{3} k_{y}}{2}\right), \\
H_{44}= & t_{0}^{44}+2 t_{1}^{44} \cos \left(k_{x}\right) \\
& +\left(3 t_{1}^{33}+t_{1}^{44}\right) \cos \left(\frac{k_{x}}{2}\right) \cos \left(\frac{\sqrt{3} k_{y}}{2}\right), \\
H_{12}= & \sqrt{3}\left(t_{1}^{11}-t_{1}^{22}\right) \sin \left(\frac{k_{x}}{2}\right) \sin \left(\frac{\sqrt{3} k_{y}}{2}\right), \\
H_{13}= & i \sqrt{3}\left(t_{1}^{14}+t_{1}^{23}\right) \cos \left(\frac{k_{x}}{2}\right) \sin \left(\frac{\sqrt{3} k_{y}}{2}\right), \\
H_{14}= & 2 i t_{1}^{14} \sin \left(k_{x}\right)+\left(-t_{1}^{14}+3 t_{1}^{23}\right) \sin \left(\frac{k_{x}}{2}\right) \cos \left(\frac{\sqrt{3} k_{y}}{2}\right), \\
H_{23}= & 2 i t_{1}^{23} \sin \left(k_{x}\right)+\left(3 t_{1}^{14}-t_{1}^{23}\right) \sin \left(\frac{k_{x}}{2}\right) \cos \left(\frac{\sqrt{3} k_{y}}{2}\right), \\
H_{34}= & -\sqrt{3}\left(t_{1}^{33}-t_{1}^{44}\right) \sin \left(\frac{k_{x}}{2}\right) \sin \left(\frac{\sqrt{3} k_{y}}{2}\right) .
\end{aligned}
$$

After converting Eq. (A1) into a new basis representation, i.e., $\left(p_{+}, d_{+}, p_{-}, d_{-}\right)$and expanding it near the $\Gamma$ point, we deduce that

$$
H_{0}(\mathbf{k})=A\left(\hat{\tau}_{z} k_{y} \hat{\sigma}_{x}-k_{x} \hat{\sigma}_{y}\right)+E_{0}+D k^{2}+\left(M_{0}-B k^{2}\right) \hat{\sigma}_{z} .
$$

Here, $A, E_{0}, D, M_{0}$, and $B$ are model parameters defined by the coupling coefficients as follows:

$$
\begin{gathered}
E_{0}=\left[t_{0}^{11}+t_{0}^{33}+3\left(t_{1}^{11}+t_{1}^{22}+t_{1}^{33}+t_{1}^{44}\right)\right] / 2, \\
D=-3\left(t_{1}^{11}+t_{1}^{22}+t_{1}^{33}+t_{1}^{44}\right) / 8, \\
A=3\left(t_{1}^{14}+t_{1}^{23}\right) / 2, \\
M_{0}=\left[t_{0}^{11}-t_{0}^{33}+3\left(t_{1}^{11}+t_{1}^{22}-t_{1}^{33}+t_{1}^{44}\right)\right] / 2, \\
B=3\left(t_{1}^{11}+t_{1}^{22}-t_{1}^{33}-t_{1}^{44}\right) / 8 .
\end{gathered}
$$

The Hamiltonian shares the same structure as the BernevigHughes-Zhang model for the $\mathrm{CdTe} / \mathrm{HgTe} / \mathrm{CdTe}$ quantum well structure. We note that $B>0$ in our system. According to the expression of the spin Chern number in Eq. (1) of the main text ( $g=0$ because there is no external magnetic field), two different phases can be distinguished by the sign of $M_{0}$. If $E(p)<E(d), M_{0}<0$, the crystal behaves like a conventional insulator, and if $E(p)>E(d)$, the crystal behaves like a QSH insulator because $M_{0}>0$.

As discussed in Ref. [3] with transverse electric states $\left[\vec{E}=\left(0,0, E_{z}\right)\right]$, the system made from YIG follows the equation for zero-energy wave functions of a nonrelativistic 
particle in a periodic vector $\left(\vec{A}=\frac{\mu^{2}-\kappa^{2}}{2 \mu} \hat{z} \times \nabla \frac{-\kappa}{\mu^{2}-\kappa^{2}}\right)$ and scalar potentials. The effective vector potential induces Zeeman-type splitting in the pseudospin states. We consider the splitting evolution under a conventional transparency region [41]. It should be noted that the introduced magnetic field breaks the symmetry from $C_{6 v}$ to $C_{6}$, which modifies the basis function written as $p_{+}, d_{+}, p_{-}$, and $d_{-}$. Then the Zeeman-type perturbation can be expressed as $H_{z}=\left(z_{p}-z_{d}\right) \hat{\tau}_{z} \hat{\sigma}_{z} / 2+$ $z_{0} I+\left(z_{p}+z_{d}\right) \hat{\sigma}_{z} / 2$ where the pseudospin splitting is $2 z_{p}$ for the $p_{ \pm}$states and $2 z_{d}$ for the $d_{ \pm}$states, $z_{0}$ is the total energy shift. It also should be noted that $z_{p}<0$ and $z_{d}>0$ in our model. We can rewrite the perturbation term in terms of Pauli matrices $\hat{\tau}$ and $\hat{\sigma}$ and consider the approximation of $z_{p} \approx-z_{d}$ as suggested by the simulated results (such an assumption will only alter the position where the topological transition occurs; it does not affect the general topological properties of the system). By defining $g=\left(z_{p}-z_{d}\right) / 2$, we obtain the new Hamiltonian as

$$
\begin{aligned}
H(\mathbf{k})= & E_{0}+z_{0}+D k^{2}+\left(M_{0}-B k^{2}\right) \hat{\sigma}_{z} \\
& +A\left(\hat{\tau}_{z} k_{y} \hat{\sigma}_{x}-k_{x} \hat{\sigma}_{y}\right)+g \hat{\tau}_{z} \hat{\sigma}_{z} .
\end{aligned}
$$

\section{APPENDIX B: CALCULATION OF THE SPIN CHERN NUMBER}

The topological invariant for the QSH effect that preserves TR symmetry is the $\mathbb{Z}_{2}$ index or the spin Chern number. It has been argued that the spin Chern number has no symmetry restrictions and can be viewed as a topological invariant in the TR-broken-QSH effect [17]. We check the Hamiltonian for a thin film of $\mathrm{Bi}_{2} \mathrm{Se}_{3}$, which can be described by the following Hamiltonian:

$$
\begin{aligned}
H_{1}(\mathbf{k})= & E_{0}+D k^{2}+\left(M_{0}-B k^{2}\right) \hat{\sigma}_{z} \\
& +\left[A\left(k_{y} \hat{\sigma}_{x}-k_{x} \hat{\sigma}_{y}\right)+\gamma U\right] \hat{\tau}_{x}
\end{aligned}
$$

where $\gamma U$ stands for a gate voltage applied between the two surfaces of the film, which breaks the TR symmetry. The spin Chern number can be evaluated by [40]

$$
C_{ \pm}= \pm\left[\operatorname{sgn}(B)+\operatorname{sgn}\left(M_{0}\right)\right] / 2,
$$

which is independent of $\gamma U$. We let $\gamma U=0$ for the convenience of the following analysis.

We perform a unitary transformation to $H(\mathbf{k})$ in Eq. (A3) and obtain $H_{S}(\mathbf{k})=S^{\dagger} H(\mathbf{k}) S$, where

$$
S=\left[\begin{array}{cccc}
1 & 0 & 0 & 0 \\
0 & 0 & 0 & 1 \\
0 & 0 & 1 & 0 \\
0 & -1 & 0 & 0
\end{array}\right] .
$$

We find an interesting mapping between the eigenvalues of $H_{1}(\mathbf{k})$ and $H_{s}(\mathbf{k})$ as well as their eigenfunctions by substituting $M_{0}$ in $H_{1}(\mathbf{k})$ with $M_{0} \pm g$. That means $E_{s}\left(M_{0}, g\right)=$ $E_{1}\left(M_{0} \pm g\right)$ and $\Psi_{s}\left(M_{0}, g\right)=\Psi_{1}\left(M_{0} \pm g\right)$, where $E$ and $\Psi$ denote the eigenvalue and corresponding eigenfunction. Since the calculation of the spin Chern number relies only on the eigenvalues and the corresponding eigenfunctions, the mapping indicates that the expression of the spin Chern number for $H(\mathbf{k})$ takes the form

$$
C_{ \pm}= \pm\left[\operatorname{sgn}(B)+\operatorname{sgn}\left(M_{0} \pm g\right)\right] / 2 .
$$

In our system, $g<0$. Increasing the magnetic field would increase $|g|$. In the initial QSH phase, both $M_{0}$ and $B$ are positive. $M_{0}-g$ is always positive regardless of the magnitude of $|g|$. The topological phase transition occurs only when $M_{0}+$ $g$ changes sign, which corresponds to the spin-up component. On the other hand, if the initial phase is a conventional insulator phase $M_{0}<0, B>0$, and the topological phase transition is only for the spin-down component when $M_{0}-g$ changes its sign. Both transitions would lead to a QAH phase characterized by the spin Chern number $C_{ \pm}=(0,-1)$.
[1] K. v. Klitzing, G. Dorda, and M. Pepper, Phys. Rev. Lett. 45, 494 (1980).

[2] F. D. M. Haldane and S. Raghu, Phys. Rev. Lett. 100, 013904 (2008).

[3] Z. Wang, Y. D. Chong, J. D. Joannopoulos, and M. Soljačić, Phys. Rev. Lett. 100, 013905 (2008).

[4] J. E. Moore, Nature (London) 464, 194 (2010).

[5] M. Z. Hasan and C. L. Kane, Rev. Mod. Phys. 82, 3045 (2010).

[6] X. L. Qi and S. C. Zhang, Rev. Mod. Phys. 83, 1057 (2011).

[7] C. L. Kane and E. J. Mele, Phys. Rev. Lett. 95, 226801 (2005).

[8] B. A. Bernevig, T. L. Hughes, and S. C. Zhang, Science 314, 1757 (2006).

[9] M. König, S. Wiedmann, C. Brüne, A. Roth, H. Buhmann, L. W. Molenkamp, X.-L. Qi, and S.-C. Zhang, Science 318, 766 (2007).

[10] C.-X. Liu, X.-L. Qi, H. J. Zhang, X. Dai, Z. Fang, and S.-C. Zhang, Phys. Rev. B 82, 045122 (2010).

[11] Y. Xu, I. Miotkowski, C. Liu et al., Nat. Phys. 10, 956 (2014).

[12] X.-L. Qi, T. L. Hughes, and S.-C. Zhang, Phys. Rev. B 78, 195424 (2008).
[13] L. Lu, J. D. Joannopoulos, and M. Soljacic, Nat. Photonics 8, 821 (2014).

[14] A. Alexandradinata, C. Fang, M. J. Gilbert, and B. A. Bernevig, Phys. Rev. Lett. 113, 116403 (2014).

[15] C.-X. Liu, X.-L. Qi, X. Dai, Z. Fang, and S.-C. Zhang, Phys. Rev. Lett. 101, 146802 (2008).

[16] F. D. M. Haldane, Phys. Rev. Lett. 61, 2015 (1988).

[17] Y. Yang, Z. Xu, L. Sheng, B. Wang, D. Y. Xing, and D. N. Sheng, Phys. Rev. Lett. 107, 066602 (2011).

[18] H. Li, L. Sheng, R. Shen, L. B. Shao, B. Wang, D. N. Sheng, and D. Y. Xing, Phys. Rev. Lett. 110, 266802 (2013).

[19] P. Wang, L. Lu, and K. Bertoldi, Phys. Rev. Lett. 115, 104302 (2015)

[20] Z. Yang, F. Gao, X. Shi, X. Lin, Z. Gao, Y. Chong, and B. Zhang, Phys. Rev. Lett. 114, 114301 (2015).

[21] X. Ni, C. He, X. C. Sun, X. P. Liu, M. H. Lu, L. Feng, and Y. F. Chen, New J. Phys. 17, 053016 (2015).

[22] S. H. Mousavi, A. B. Khanikaev, and Z. Wang, Nat. Commun. 6, 8682 (2015).

[23] A. B. Khanikaev, R. Fleury, S. H. Mousavi, and A. Alu, Nat. Commun. 6, 8260 (2015). 
[24] M. Hafezi, E. A. Demler, M. D. Lukin, and J. M. Taylor, Nat. Phys. 7, 907 (2011).

[25] G. Q. Liang and Y. D. Chong, Phys. Rev. Lett. 110, 203904 (2013).

[26] W.-J. Chen, S.-J. Jiang, X.-D. Chen, B. Zhu, L. Zhou, J.-W. Dong, and C. T. Chan, Nat. Commun. 5, 5782 (2014).

[27] K. Y. Bliokh, D. Smirnova, and F. Nori, Science 348, 1448 (2015).

[28] A. B. Khanikaev, S. Hossein Mousavi, W.-K. Tse, M. Kargarian, A. H. MacDonald, and G. Shvets, Nature Mater. 12, 233 (2013).

[29] T. Ma, A. B. Khanikaev, S. H. Mousavi, and G. Shvets, Phys. Rev. Lett. 114, 127401 (2015).

[30] L.-H. Wu and X. Hu, Phys. Rev. Lett. 114, 223901 (2015).

[31] C. He, X.-C. Sun, X.-P. Liu, M.-H. Lu, Y. Chen, L. Feng, and Y.-F. Chen, Proc. Natl. Acad. Sci. U.S.A. 113, 4924 (2016).
[32] C. He, X. Ni, H. Ge, X.-C. Sun, Y.-B. Chen, M.-H. Lu, X.-P. Liu, and Y.-F. Chen, Nat. Phys. 12, 1124 (2016).

[33] J. Mei, Z. Chen, and Y. Wu, Sci. Rep. 6, 32752 (2016).

[34] L.-H. Wu and X. Hu, Sci. Rep. 6, 24347 (2016).

[35] E. Prodan, Phys. Rev. B 80, 125327 (2009).

[36] D. M. Pozar, Microwave Engineering (Wiley, Hoboken, NJ, 2004).

[37] K. Sakoda, Opt. Express 20, 3898 (2012).

[38] A. V. Nalitov, D. D. Solnyshkov, and G. Malpuech, Phys. Rev. Lett. 114, 116401 (2015).

[39] Z.-G. Chen and Y. Wu, Phys. Rev. Appl. 5, 054021 (2016).

[40] H. Li, L. Sheng, D. N. Sheng, and D. Y. Xing, Phys. Rev. B 82, 165104 (2010).

[41] A. R. Davoyan and N. Engheta, Phys. Rev. Lett. 111, 257401 (2013). 